\title{
Construction and Simulation on Control System of Voice Coil Linear Motor Based on PCC
}

\author{
Rongrong $\mathrm{Wu}$ \\ School of Electronic Engineering, Tianjin University of \\ Technology and Education, \\ Tianjin 300222, China \\ 519564315@qq.com \\ Shengli Lu \\ Engineering Training Center, Tianjin University of \\ Technology and Education, \\ Tianjin 300222, China \\ LSL5612@163.com
}

\author{
Liqiang Wang \\ School of Electronic Engineering, Tianjin University of \\ Technology and Education, \\ Tianjin 300222, China \\ 915825202@qq.com \\ Guangwei Wang \\ School of Electronic Engineering, Tianjin University of \\ Technology and Education, \\ Tianjin 300222, China \\ 532119472@qq.com
}

\begin{abstract}
On the basis of establishing control system model of voice coil linear motor, saturated link and dead zone link of nonlinear systems are made Simulink simulation to implement the optimization of control system. B\&R Automation Studio integrated development environment is used to automatically generate the $C$ code, which can be downloaded to run on the B\&R PCC, and the main performance index of control system achieve the design requirements.
\end{abstract}

Keywords- Voice Coil Linear Motor; Saturated Link; Dead Zone Link; Automation Studio; B\&R PCC;

\section{INTRODUCTION}

Linear motor's acceleration can be up to $10 \mathrm{~g}$ or more after direct drive. It is more suitable for high speed, high acceleration, high precision positioning, and speed drive. It is an ideal choice that linear motor is used in the high speed reciprocating linear motion occasions. In high efficiency requirement areas, such as machine and the field of medical devices, the use of linear motor can greatly improve the production efficiency [1].

Automation Studio (AS) of B\&R integrates with the MATLAB/Simulink simulation facility, and can automatically generate $\mathrm{C}$ code, which is downloaded to the B\&R PCC to run fro $m$ the control system simulation model.

AS integrated development environment based on $\mathrm{B} \& \mathrm{R}$ can efficiently complete control system design based on B\&R PCC and ensure the main indicators of control system designed to meet the requirements [2]. It is undoubtedly a very good design choice for voice coil linear motor control system.

\section{THE PROGRAMMING SOFTW ARE AS}

Automation Studio is an integrated programming environment for $\mathrm{B} \& \mathrm{R}$ automation products, it provides users with many programming language spaces and a lot of diagnostic tools. Automation studio software packages design for every object (logic, motion, measurement, communication, display) of the machine and processes (configuration, project planning and management, diagnostics, debugging and maintenance). The design of the controller, servo, Safety technology and communications can implement in the "ALL in One" Automation Studio toolkit for B\&R Automation Studio. Automation Studio provides a complete platform engineering design and application for the system integrated a variety of control de mands such as motor, it makes the code generation, remote diagnostics and maintenance are integrated as a whole. B\&R Automation Studio's function is shown in Fig. 1.

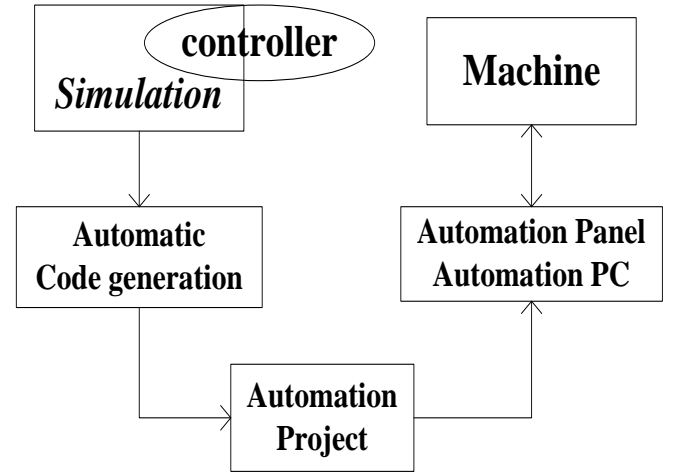

Figure 1. B\&R Automation Studio's functional schematic.

\section{THE VOICE COIL LINEAR MOTOR}

A. The mathematical model of Voice Coil Motor

The mathematical model of the voice coil motor is established according to the work dynamic characteristics of the voice coil motor [3]. If you want to design a voice coil motor control system, which has a good position and speed control of voice coil motor, the 
first problem is to establish a reasonable mathematical model of the voice coil motor. The mathematical model can analyze the influence of various parameters on the dynamic performance of the voice coil motor and provide the theoretical basis for the selection parameters and the establishment of control system for the design of the voice coil motor. Mathematical mode is established using the voltage equation and stress equations, according to the electromagnetic theory, based on the simplified model of voice coil motor [4].

According to the electromagnetic theory, when the rotor coil is moving, in addition to the electromagnetic force, the coil winding can cut magnetic lines, so also anti electric $E_{a \text { is generated, its size is }}$

$$
E_{a}=B_{\delta} L V
$$

Signal source voltage of the voice coil motor, namely armature voltage, is assumed to be $U_{a}$, the resistance of the coil and the circuit resistance constitute the armature circuit resistance, When the voltage balance equation of steady state is based on Kirchhoff's voltage law can write

$$
U_{a}=R_{a} I_{a}+E_{a}=R_{a} I_{a}+B_{\delta} L V
$$

In the control system, the voice coil motor is always in dynamic condition, because the armature existing inductance $L_{a}$ and armature current $i_{a}$ are constantly changing, so the armature coil generates a voltage $L_{a} \frac{d i_{a}}{d t}$, the voltage balance equation of dynamic as follows

$$
u_{a}=R_{a} i_{a}+\ell_{a}+L_{a} \frac{d i_{a}}{d t}=R_{a} i_{a}+B_{\delta} L v+L_{a} \frac{d i_{a}}{d t}
$$

Under the dynamic conditions, the mover of the voice coil motor often work on accelerating and decelerating state, to accelerate the completion of linear motion, so the electromagnetic force required to overcome the inertia force $f_{m}$ of moving part, The inertial force $f_{m}$ is

$$
f_{m}=m a=m \frac{d v}{d t}=m \frac{d^{2} x}{d t^{2}}
$$

In the formula, $m$ is a dynamic quality parts, $v$ is a dynamic motion velocity, $x$ is a linear displacement of the rotor motion.

Dynamic exercise includes the inertia force and friction force $f_{c}$, and friction includes two kinds, dry friction and viscous friction. Which played a major role in the viscous friction, its size is much larger than that of dry friction, and researchers consider only the blocking effect of viscous friction. The viscous friction force depends on the size of the moving coil motor speed, which is proportional to the speed, the size is

$$
f_{c}=c v=c \frac{d x}{d t}
$$

In the formula, $\mathrm{c}$ is the viscous damping coefficient.

The voice coil motor rotor coil is in motion, the electromagnetic force $f_{\ell}$, its size is

$$
f_{e}=B_{\delta} L i_{a}
$$

There are inertial force $f_{m}$ and viscous friction $f_{c}$ when the motor is in motion. According to the relationship between these forces, stress equation of the voice coil motor in dynamic is

$$
f_{\ell}=m a+c v=m \frac{d^{2} x}{d t^{2}}+c \frac{d x}{d t}
$$
can be

The type (1), (3), (6), (7) for the Laplace transform

$$
\ell_{a}(s)=B_{\delta} L v(s)
$$

$$
\begin{aligned}
& R_{a} i_{a}(s)+\ell_{a}(s)+s L_{a} i_{a}(s) \\
& =R_{a} i_{a}(s)+B_{\delta} L V(s)+s L_{a} i_{a}(s) \\
& =u_{a}(s)
\end{aligned}
$$

$$
f_{\ell}(s)=B_{\delta} L i_{a}(s)
$$

$$
f_{\ell}(s)=m a(s)+c v(s)=m s^{2} x(s)+c s x(s)
$$

By (8), (9), (10), (11) to get the transfer function between the voice coil motor armature voltage and displacement. The transferfunction is

$$
\begin{aligned}
& G(S)=\frac{X(s)}{u_{a}(s)} \\
& =\frac{B_{\delta} L}{L_{a} m S^{3}+\left(L_{d} c+R_{d} m\right) S^{2}+\left(R_{a} c+B_{\delta}^{2} L^{2}\right)_{S}}
\end{aligned}
$$

According to the transfer function, the mathematical model of the voice coil motor is shown in Fig. 2. 


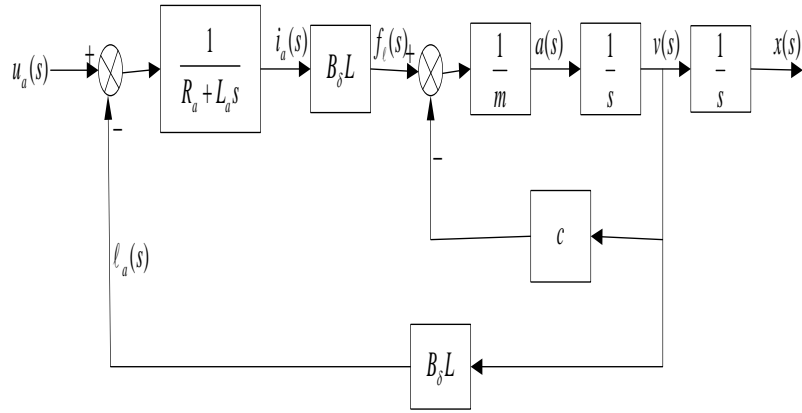

Figure 2. Block diagram of voice coil motor.

B. The nonlinear analysis and simulation of voice coil linear motor control system model

Simu link tools in MATLAB software package itself have nonlinear module, which can easily analysis and research for any nonlinear high system[5].

In this section, focuses on saturated links and dead zone in nonlinear system with SIMULINK simulation, through the simulation analysis to implement the optimization of voice coil linear motor control system. Different nonlinear link (saturation, dead band) are introduced in current loop, speed loop and position loop of the voice coil linear motor control system, by looking at the step response of system, to analyze and compare different nonlinear link will affect the performance of the system.

Voice coil linear motor control system model is established to use Simulink toolbox in MATLAB [6]. As shown in Fig. 3, Fig. 4 and Fig. 5 respectively are threering system block diagram of voice coil linear motor control systemwithout any nonlinear part.

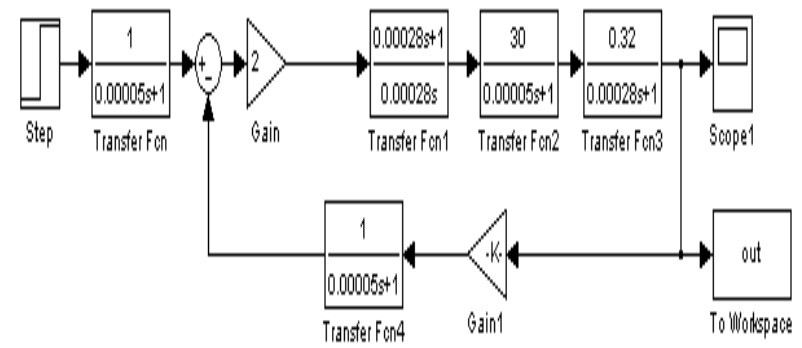

Figure 3. Current loop system block diagram.

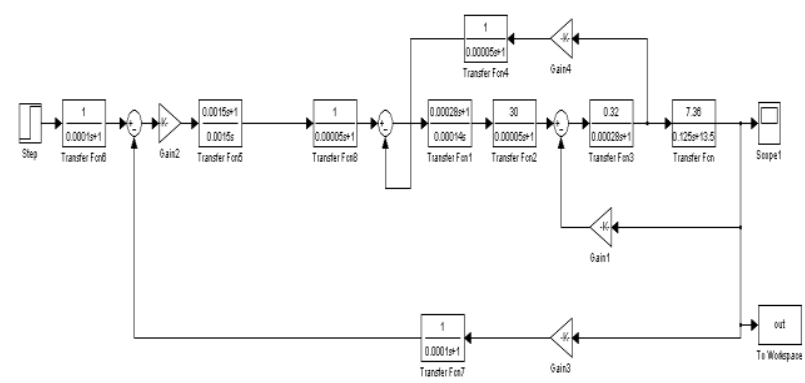

Figure 4. Velocity loop system block diagram.

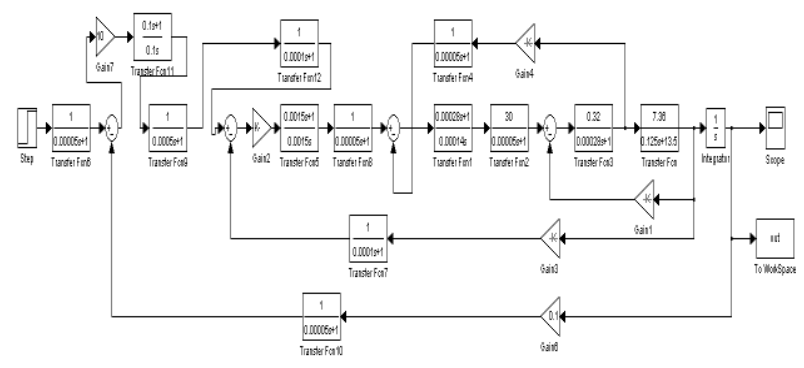

Figure 5. Position loop system block diagram.

1) Analysis and simulation of Saturated nonlinear

Saturation widely exist in all kinds of control systems, such as actuators or some limiter for security and artificial. Their presence or intervention can make originally linear systems to nonlinear, to change the ess ence characteristic of the system.

By adding saturated nonlinear system link, the system step response is compared when the output upper and lower limits of saturated nonlinear link changed, then recording the results of many times simulation to the workspace by using the To Workspace module in the Simulink, and drawing on the same image.

As shown in Fig. 6, Fig. 7 and Fig. 8, they are respectively three-ring system block diagrams for voice coil linear motor control system with Saturated nonlinear.

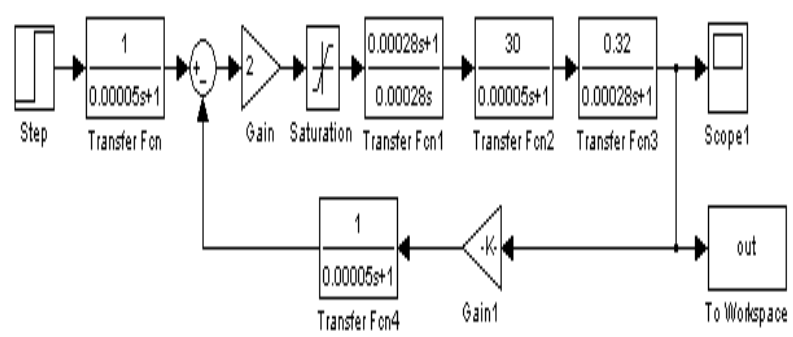

Figure 6. Current loop system block diagram of saturated nonlinear.

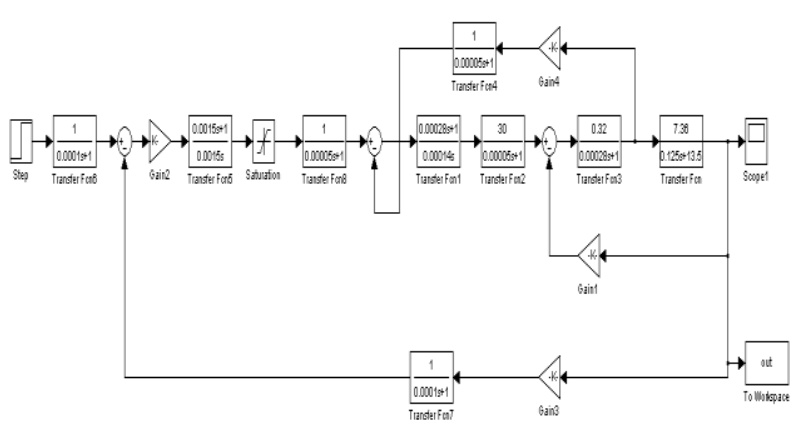

Figure 7. Velocity loop system block diagram of sat urated nonlinear. 


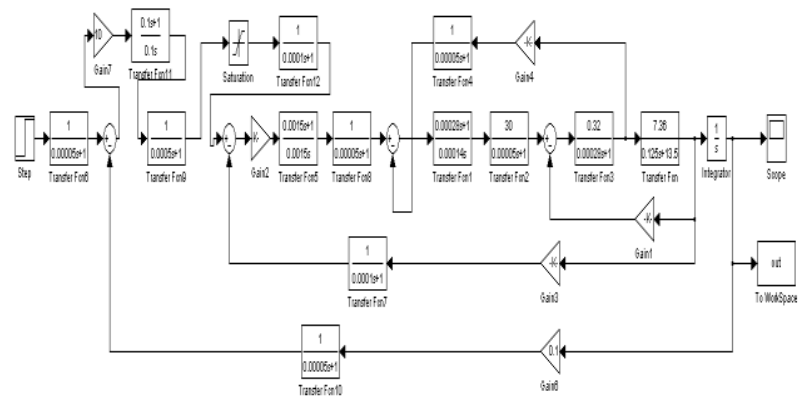

Figure 8. Position loop system block diagram of saturated nonlinear.

In the case of undistorted current loop step response system, the output upper limit of the saturation nonlinear part is set at 1.75 , and the output lower limit is -1.75 , the simulation results are recorded into the workspace variable out 1 ; the upper limit is 1.875 , the lower limit is -1.875 , the simulation results are recorded into the works pace variable out 2 ; the upper limit up to 2 , the lower limit is -2 , the simulation results are recorded into the works pace variable out 3 ; the upper limit is 2.125 , the lower limit is -2.125 , the simulation results are recorded into the workspace variable out4; the upper limit is 2.25 , the lower limit is -2.25 , the simulation results are recorded into the workspace variable out5. Simulation results of above five different output ranges are shown in Fig. 9.

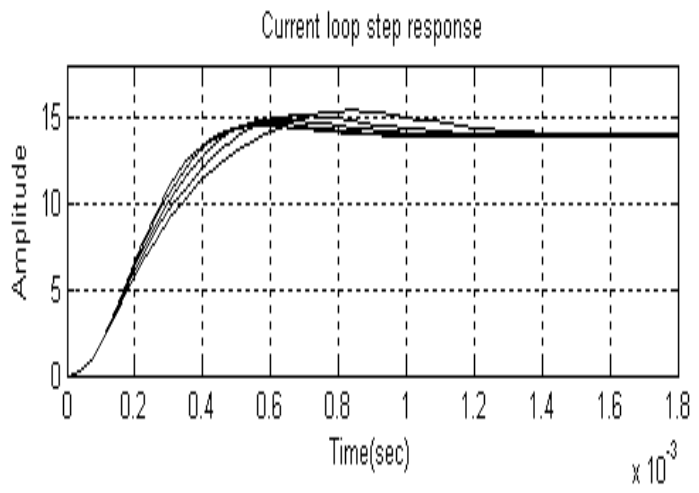

Figure 9. Current loop step response of saturated nonlinear.

In the case of undistorted speed loop step response system, the output upper limit of the saturation nonlinear part respectively are $30,40,50,60$ and 70 , corresponding to the output lower limit are $-30,-40,-50$, -60 and -70 . Simulation results of above five different output ranges are shown in Fig. 10.

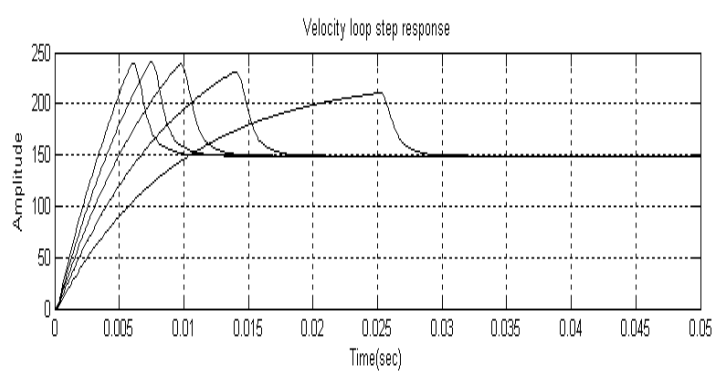

Figure 10. Velocity loop step response of saturated nonlinear.
In the case of undistorted position loop step response system, the output upper limit of the saturation nonlinear part respectively are $1.5,2.125,2.75,3.375$ and 4 , corresponding to the output lower limit are $-1.5,-2.125$, $-2.75,-3.375$ and -4 . Simulation results of above five different output ranges are shown in Fig. 11.

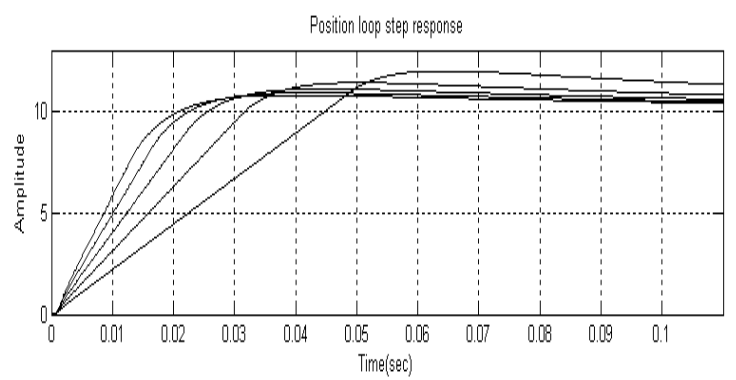

Figure 11. Position loop step response of saturated nonlinear.

Therefore, the added saturated part of the system will have some impact on the system from Fig. 9, Fig. 10 and Fig. 11: when the output range of saturation nonlinear aspects is narrow, step response speed of the system is slow and the rising time is long. At the same time, overshoot is small and oscillation is not obvious; with the expansion of the output range, accelerating the response speed of the system and reducing greatly the rise time, the system is also associated with significant oscillations. The reason is that the saturated part will play a limiting action on the signal. It is easy to imagine that the restriction is stronger, the output of the system is not easy to overshoot and the response becomes slower [7].

2) Analysis and Simulation of Dead zone nonlinear

Dead is also known as a dead zone. Some measurement, the conversion parts and a variety of amplifiers of control systems have often the dead zone in the vicinity of zero. In addition, due to the motor shaft has a static friction, the voltage applied to the armature must reach a certain value (no-load starting voltage), then the motor can begin rotating, so the motor has a dead zone (no-load starting voltage is the insensitive range of motor).

By adding dead zone nonlinear system link, the system step response is compared when the output upper and lower limits of dead zone nonlinear link changed, then recording the results of many times simulation to the workspace by using the To Workspace module in the Simulink, and drawing on the same image. As shown in Fig. 12, Fig. 13 and Fig. 14 respectively are three-ring system block diagrams of voice coil linear motor control systemwith dead zone nonlinear. 


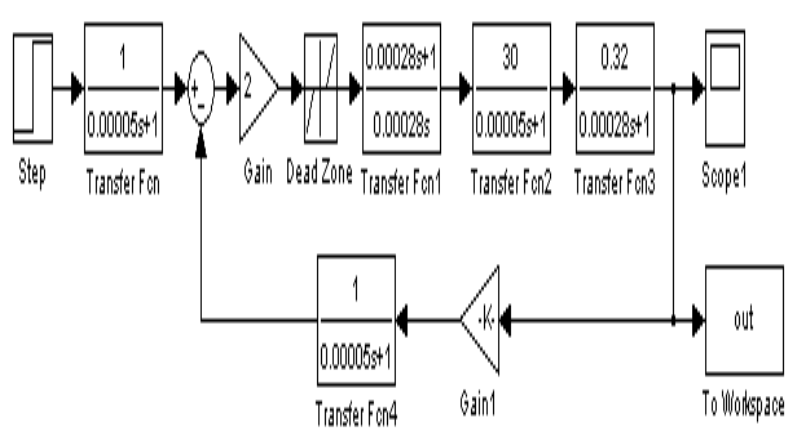

Figure 12. Current loop system block diagram of dead zone nonlinear.

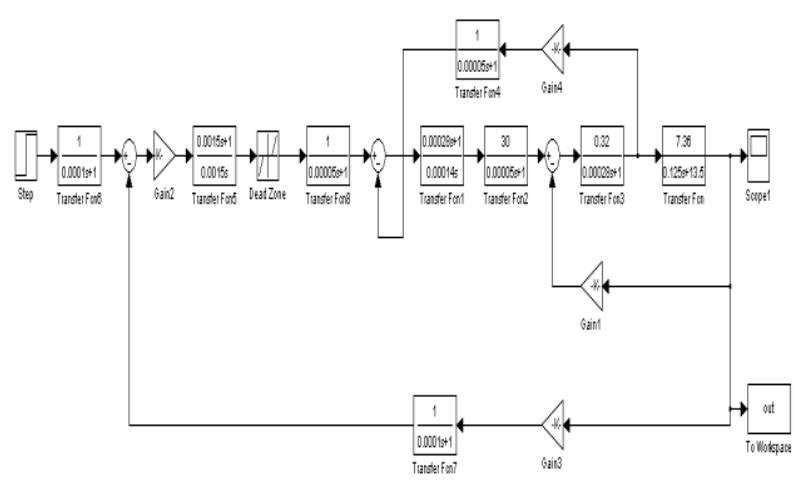

Figure 13. Velocity loop system block diagram of dead zone nonlinear.

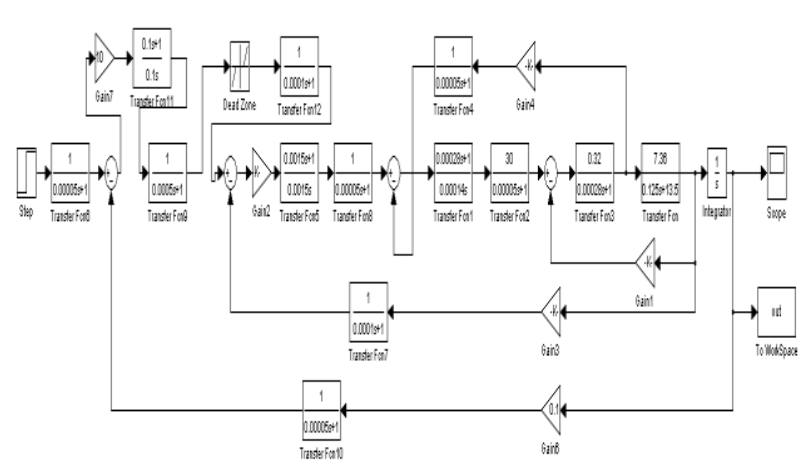

Figure 14. Position loop system block diagram of dead zone nonlinear.

In the case of undistorted current loop step response system, the output upper limit of the dead zone nonlinear part respectively are $0.001,0.005,0.01,0.05$ and 0.1 , corresponding to the output lower limit are -0.001 , $0.005,-0.01,-0.05$ and -0.1 . Simulation results of above five different output ranges are shown in Fig. 15.

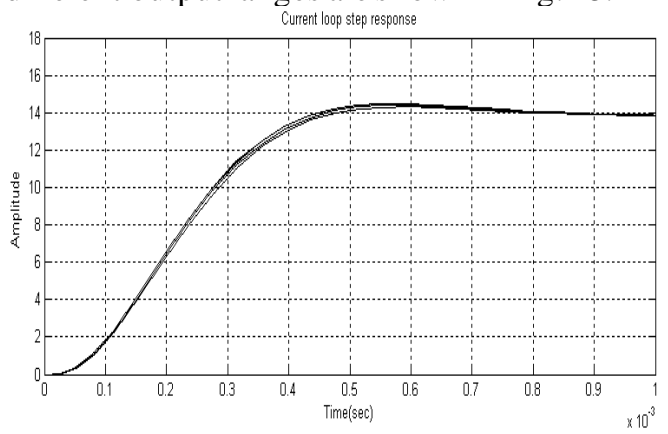

Figure 15. Current loop step response of dead zone nonlinear.
In the case of undistorted Velocity loop step response system, the output upper limit of the dead zone nonlinear part respectively are 1, 30, 60, 90 and 120, corresponding to the output lower limit are $-1,-30,-60,-$ 90 and -120 . Simulation results of above five different output ranges are shown in Fig. 16.

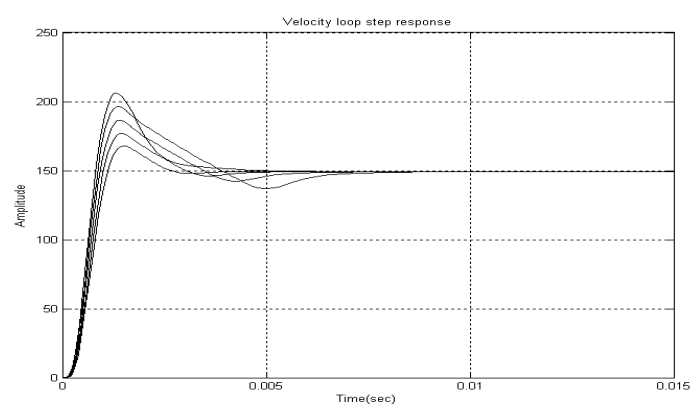

Figure 16. Velocity loop step response of dead zone nonlinear.

In the case of undistorted Position loop step response system, the output upper limit of the dead zone nonlinear part respectively are $0.00001,0.0001,0.001,0.01$ and 0.1 , corresponding to the output lower limit are -0.00001 , $-0.0001,-0.001,-0.01$ and -0.1 . Simulation results of above five different output ranges are shown in Fig. 17.

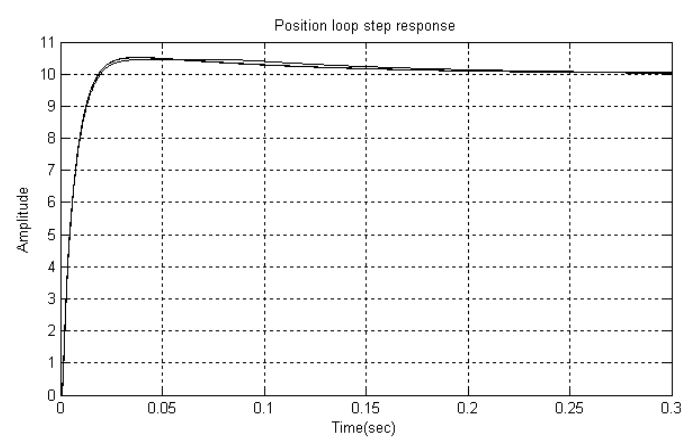

Figure 17. Position loop step response of dead zone nonlinear.

As researchers can see that the time of system begins to respond to a step input signal is gradually delayed with the increase of the dead band range from figure 15 , 16 and 17 . The reason is dead links will ignore the input in the dead zone, making response of the system slow [8].

\section{Aut OMATiCALly GenERATE C CODE}

$B \& R$ programming software Automation Studio integrates Simulink model code translation function, it can translate the existing Simulink model into PLC controller available programming language code, and can achieve functions by other means impossible or require a lot of time to achieve [9].

First, Installing simulation tool interface provided by $B \& R$ in MATLAB software interface. After installation, there appears B\&R Automation Studio Toolbox in the Simu link Library catalog. The B\&R Automation Studio Toolbox catalog includes $\mathrm{B} \& \mathrm{R}$ set block, $\mathrm{B} \& \mathrm{R}$ input block, B\&R output block, B\&R parameter block etc. It is shown in Fig. 18. 


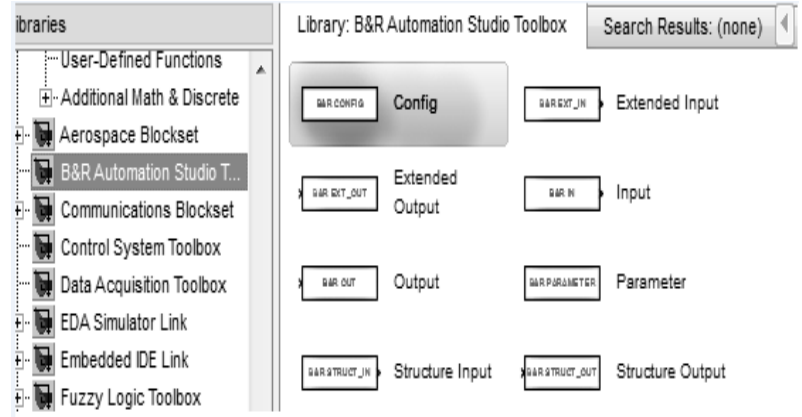

Figure 18. B\&R Toolbox module.

These function blocks instead of the original modules will be added to the motor control system simulation model. Step signal changes for input module. PID parameters changes for Parameter module that it is good for real-time adjustment parameters in the PLC. Output module changes for output signal. It is shown in Fig. 19.

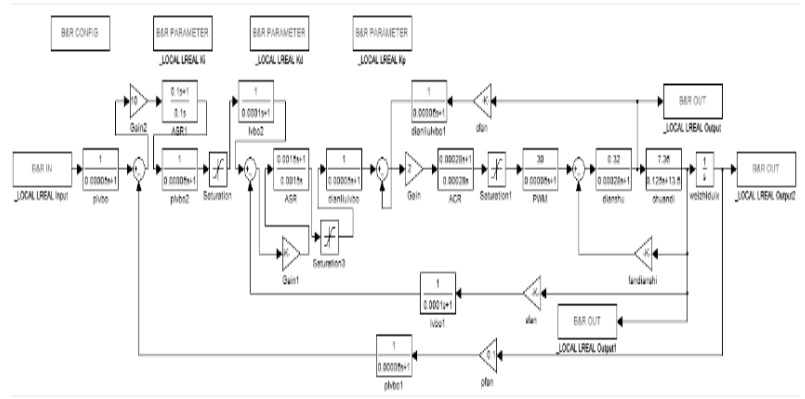

Figure 19. The simulation model joining B\&R Simulation Module.

Then, the modeling and simulation is completed in MATLAB/Simulink, opening the B\&R programming software Automation Studio to configure a series of parameters of MATLAB and AS. As shown in Fig. 20 is the location parameter setting. As shown in Fig. 21 is the time parameter setting.

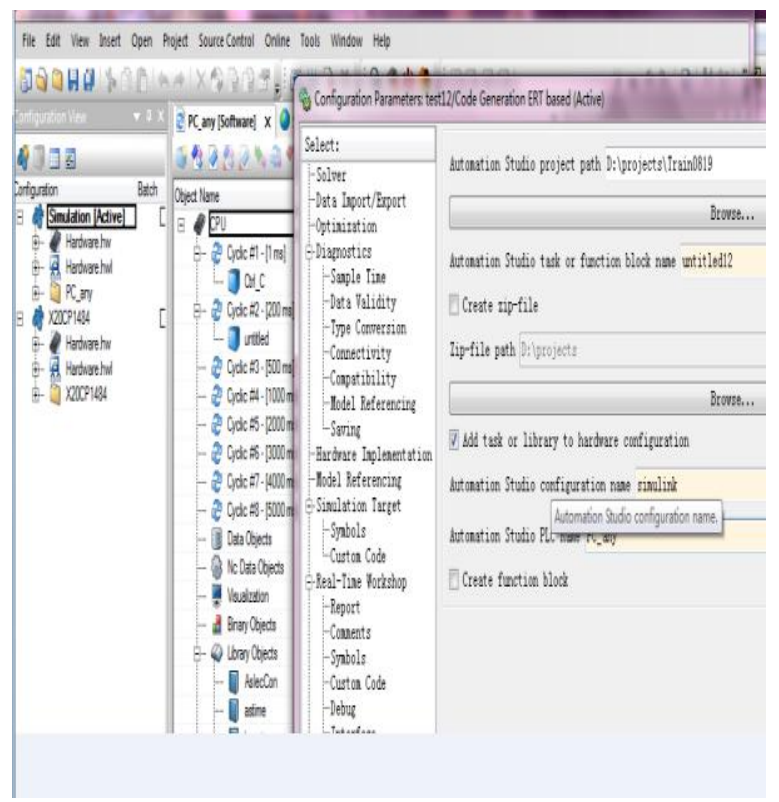

Figure 20. The location parameter setting.

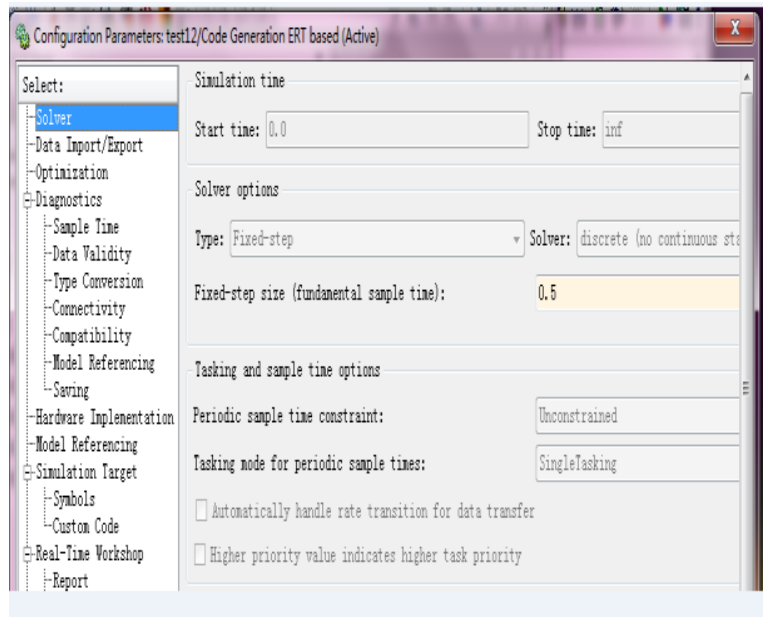

Figure 21. The time parameter setting.

Finally, to start the $\mathrm{C}$ code generation button, the $\mathrm{C}$ code will be automatically generated and successfully implanted into the programming environment of AS project. Further, "Brsystem" and "sys_lib" are added to the project file, then process will be downloaded to the PCC hardware of B\&R by AS, the voice coil linear motor control system can run in the B\&R motion control system [10]. As shown in Fig. 22 is the actual running position of linear motor. Researchers can see that the velocity of motor which running according to fixed law is high-speed and stable from the figure

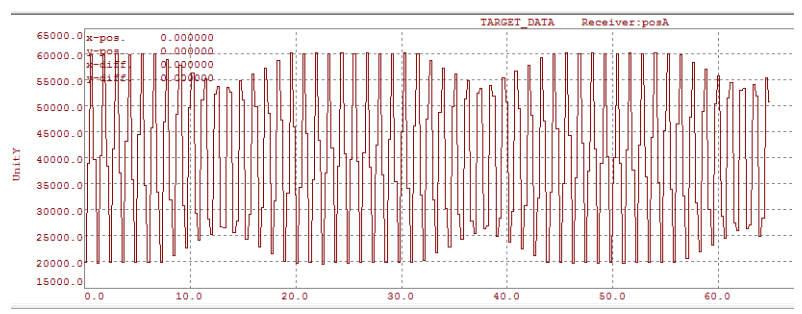

Figure 22. The actual running positon of linear motor.

\section{CONCLUSIONS}

Through the analysis of the structure, mathematical model of voice coil linear motor control system is established and the nonlinear system simulation in Simulink is made. Due to its particularity, there is no common research methods of nonlinear system, but Simulink can easily complete the modeling and simulation of nonlinear system, and provides the foundation for further correction and complex system.

In addition, it can automatically generate $\mathrm{C}$ code after generating $\mathrm{B} \& \mathrm{R}$ Toolbox by $\mathrm{B} \& \mathrm{R}$ Automation Studio Target for Simulink, and then imported into the $\mathrm{B} \& \mathrm{R}$ programming environment AS. The existing Simulink model can be transplanted to the PLC hardware to use, algorithm and objects be proved on the actual hardware objects. This enables staff which already accustomed to using MATLAB/Simulink simulation no longer work as before complicated work. This can greatly improve the programming efficiency and reduce development costs, meanwhile avoid programming errors. 


\section{REFERENCES}

[1] Xi Zhou. The key technology research of high frequency voice coil linear motor motion system [D]. Tianjin: Tianjin University of Technology and Education,2013

[2] Rong Qi, Weirong Xiao. Programmable computer control technology [M]. Beijing: Electronic Industry Press,2009

[3] Zhongxian Wang. MATLAB Modeling and Simulation Application [M]. Beijing: Machinery Industry Press, 2010

[4] Lili Liu. Research on Position Servo Control System for Voice Coil Motor[D]. Harbin: Harbin Institute of Technology,2010

[5] Hui Zhang, Jiang Liu, Guangfeng Guan et al. Approaches to Non-linearity Compensation of Servo Proportional Valve [J].Machine Tool \& Hydraulics, 2010, 38(11):1-3
[6] Serkies P, Orlowska-Kowalska T, Cychowski M, et al. Robust model predictive speed control of the drive system with an elastic joint $[\mathrm{C}] / /$ EUROCON-International Conference on Computer as a Tool(EUROCON), 2011 IEEE. IEEE, 2011: 1-4

[7] Guolai Yang, Mei Zheng, Guixiang Bai. Emulation of Saturation of Non-linear System Based on SIMULINK [J]. Journal of Gansu Sciences, 201 1,23(4):90-92

[8] Li Zhao. Emulation of Dead zone of Non-linear System Based on SIMULINK [J]. Value Engineering, 2010,(3):85

[9] Lei Li, Ziyu Huang Gang Li. Simulink PLC Coder in the B \& R motion Control System Application [J]. Instrumentation Technology, 2011, 6:59-61

[10] Xin Jin. Design and implementation of multi mass elastic torsion system controller based on PCC. Shanghai: Donghua University,2010. 